\title{
Hierarchical Analysis of Variation in the Mitochondrial 16SrRNA Gene among Five Different Insect Orders
}

\author{
Chatterjee Susmita \\ Division of Genetics, Indian Agricultural Research Institute, New Delhi, India \\ Email: susmita.chatterjee1@gmail.com
}

Received 19 January 2015; accepted 22 November 2015; published 25 November 2015

Copyright (C) 2015 by author and Scientific Research Publishing Inc.

This work is licensed under the Creative Commons Attribution International License (CC BY). http://creativecommons.org/licenses/by/4.0/

\begin{abstract}
Nucleotide sequences from a $500 \mathrm{bp}$ region of the 16SrRNA gene were analyzed for ten insect pests of five different orders to examine the patterns of variation within the gene fragment and the taxonomic levels for which it showed maximum utility in phylogeny estimation. A hierarchical approach was adopted in the study through comparison of levels of sequence variation among taxa at different taxonomic levels. Among them, partial 16SrRNA gene was amplified in ten insects of five different orders. As previously reported for many holometabolous insects, the 16SrRNA gene data is reported here for 5 different orders were highly AT-rich and exhibited strong siteto-site variation in substitution rate. The partial 16SrRNA genes of five out of ten insects were reported first time. Primers were made from blasting 2 different genera of the order Diptera. These primers were proven to be universal as it amplified the partial 16SrRNA gene in ten different insects across five different orders, Diptera, Coleoptera, Heteroptera, Lepidoptera and Hymenoptera. Later, a phylogenetic tree was also constructed for understanding and analyzing the relation of above five orders. This study resulted in unusual findings which were as follows: All the species of Drosophila of order Diptera were evolutionary more closely related to Dysdercus koenigii of order Heteroptera than Bactrocera cucurbitae of Drosophilan order, Diptera in terms of partial 16SrRNA gene sequence. Similarly, Spodoptera litura and Helicoverpa armigera belonged to same family Noctuidae whereas Pieris brassicae belonged to family Pieridae. All belonged to order Lepidoptera. The results showed that Spodoptera litura in terms of partial 16SrRNA gene sequence was evolutionary more close to Pieris brassicae than Helicoverpa armigera.
\end{abstract}

\section{Keywords}

16SrRNA Gene, PCR, Amplification, Phylogenetic Tree 


\section{Introduction}

Drosophila is a model organism for research study for a longtime. However, other insects, especially pests which have a great significant value in agriculture were rarely studied. Selecting a gene for phylogenetic analysis required matching the level of sequence variation to the desired taxonomic level of study. The mitochondrial DNA of mammals had been used for molecular evolution studies [1] and similar techniques had been applied to insects as well [2]-[5]. Because of its high rate of evolution, mitochondrial DNA had been extremely useful molecule for high resolution analysis of evolutionary processes [6]. It had been used in the phylogenetic analysis of insects [7]. Factors other than the distribution of rate variation among sites could determine the shape of the sequence divergence accumulation curve. For instance, in many holometabolous insects, including Hymenoptera and Diptera, mitochondrial DNA exhibited a nucleotide composition which is strongly biased toward adenine and thymine (AT bias). For some groups, the mean percentage of AT could be higher than 80\% [8]-[9]. When the base composition is biased to that degree, obviously, the ratio of transversions (tv) to transitions (ti) increased. These led to further reduce the ability to correctly estimate the number and proportion of hidden mutations and, hence, it also reduced the ability to correct sequence divergence for hidden changes. 16SrRNA of mitochondrial genome holds a crucial role in the mRNA translation and remained highly conserved throughout the evolutionary process. Here, 16SrRNA gene was selected for study across the insect orders. Primers were constructed by blasting two different genera of Diptera and were used for amplification in insect pests belonging to Diptera, Coleoptera, Heteroptera, Lepidoptera and Hymenoptera. Later, a phylogenetic tree was also constructed for understanding and analyzing the relation of five above orders.

\section{Materials and Methods}

\subsection{Sources of Sequence Data}

For 16S ribosomal partial gene sequences, I blasted Drosophila melanogaster partial 16S ribosomal RNA gene sequence (GenBank: X53506.1) and Bacterocera cucerbitae partial 16S ribosomal RNA gene sequence (GenBank: FJ168025.1). These were blasted in NCBI blast tool in Fasta format to obtain the conserved regions of $16 \mathrm{~S}$ ribosomal RNA partial gene. Gene-specific primer used for amplification of the target partial gene was designed in-house manually. The sequence specificities of the primer sequence so designed was verified using the BLAST program available at the NCBI website (www.ncbi.nlm.nih.gov/BLAST/). The primer sequence was also evaluated for various other characteristics like melting point, presence of secondary structure formations like hairpins and propensity for dimer formation using software available in internet, Oligonucleotide Properties Calculator (http://www.basic.northwestern.edu/biotools/oligocalc.html).

\subsection{Isolation of DNA}

DNA was extracted from ten different insect species of five different orders (Table 1) by using Qiagen tissue kit and was verified by running in $1 \%$ agarose gel and then quantified by Spectramax M5, a dual-monochroma- tor and further processed for partial 16SrRNA gene amplification.

The PCR was carried out using Taq DNA polymerase (Fermentas) with the following general conditions: 15 $20 \mathrm{Ng}$ of genomic DNA was used in a $20 \mu \mathrm{l}$ reaction with $5 \mathrm{U} / \mu \mathrm{l}$ of Taq DNA polymerase, $2.5 \mathrm{~mm}$ each dNTP mix and $10 \mathrm{pM} / \mu \mathrm{l}$ of each primer (Table 2) with the following condition.

Table 1. Following species were considered for study.

\begin{tabular}{cl}
\hline Order & \multicolumn{1}{c}{ Name of Species } \\
\hline Diptera & Drosophila ananesse, Drosophila jambulina, Drosophila melanogaster, Bacterocera cucerbitae \\
Coleoptera & Coccinella septempunctata \\
Heteroptera & Dysdercus koenigii \\
Lepidoptera & Spodoptera litura, Helicoverpa armigera, Pieris brassicae \\
Hymenoptera & Apis mellifera \\
\hline
\end{tabular}


Table 2. Primers used for gene study.

\begin{tabular}{ccccc}
\hline Gene Name & Primer Name & Primer Sequence $\left(5,-3{ }^{\prime}\right)$ & $\operatorname{Tm}\left({ }^{\circ} \mathrm{C}\right)$ & $\begin{array}{c}\text { Targeted } \\
\text { Region (bp) }\end{array}$ \\
\hline \multirow{2}{*}{ 16SrRNA } & Forward & TAAAGTCTGACCTGCCCACTGAAT & 56 & \multirow{2}{*}{500} \\
& Reverse & CTTAATCCAACATCGAGGTCGCAA & 56 & \\
\hline
\end{tabular}

Following an initial denaturation at $94^{\circ} \mathrm{C}$ for $5 \mathrm{~min}, 37$ cycles at $94^{\circ} \mathrm{C}$ were performed, each annealing at $59^{\circ} \mathrm{C}$ for $45 \mathrm{~s}$ and extension at $72^{\circ} \mathrm{C}$ for $30 \mathrm{~s}$. A final extension was run at $72^{\circ} \mathrm{C}$ for $5 \mathrm{~min}$. The PCR products $(5 \mu \mathrm{l})$ were resolved in $1 \%$ agarose gel which was run at $80 \mathrm{~V}$ for 45 minutes. Negative PCR control was also run with double distilled water instead of DNA to eliminate doubts. The amplified products were eluted from the gel and purified by gel purification kit before sending for sequencing. The sequencing both through forward and reverse primers were done by Sanger method through outsourcing.

The gene sequences obtained from the samples were aligned through clustal W software in www.genome.jp/tools/clustalw/ and similarities and dissimilarities were assessed.

\subsection{Construction of Phylogenetic Tree}

Based on homology and genetic dissimilarities, a phylogenetic tree was constructed using rooted and branched clustal W software, www.genome.jp/tools/clustalw/.

Finally the sequences were submitted to NCBI database in www.ncbi.nlm.nih.gov/.

\section{Results and Discussion}

Bands amplified (Figure 1) have been verified to be ribosomal DNA by direct sequencing of the PCR products and blasting the obtained sequences with NR sequences of the NCBI database. The sequences were obtained both by forward and reverse primers and then verified and finally submitted to the NCBI database. In Coccinella septempunctata, Dysdercus koenigii, Spodoptera litura, Helicoverpa armigera and Pieris brassicae, this partial gene was reported first time. The sequences were published in NCBI database and have gene bank accession numbers (Table 3). The versatility of these ribosomal primers in insects was clearly demonstrated. Experimental studies confirmed the theoretical expectations based on sequence conservation about the relative performance of the forward and reverse primers. Since these primers amplified different orders, The identity score of all the sequences with each other were as follows: 1. Drosophila ananesse; 2. Drosophila melanogaster; 3. Drosophila jambulina; 4. Bactrocera cucurbitae; 5. Coccinella septempunctata; 6. Dysdercus koenigii; 7. Spodoptera litura; 8. Helicoverpa armigera; 9. Pieris brassicae; 10. Apis mellifera.

Sequences (1:2) Aligned. Score: 90.7834

Sequences (1:3) Aligned. Score: 95.0521

Sequences (1:4) Aligned. Score: 76.0925

Sequences (1:5) Aligned. Score: 68.0782

Sequences (1:6) Aligned. Score: 88.4106

Sequences (1:7) Aligned. Score: 67.8378

Sequences (1:8) Aligned. Score: 68.2266

Sequences (1:9) Aligned. Score: 69.4511

Sequences (1:10) Aligned. Score: 60.8466

Sequences (2:3) Aligned. Score: 86.9792

Sequences (2:4) Aligned. Score: 74.2931

Sequences (2:5) Aligned. Score: 67.7524

Sequences (2:6) Aligned. Score: 96.0265

Sequences (2:7) Aligned. Score: 64.3243

Sequences (2:8) Aligned. Score: 65.5172

Sequences (2:9) Aligned. Score: 68.9737

Sequences (2:10) Aligned. Score: 60.0529

Sequences (3:4) Aligned. Score: 70.0521

Sequences (3:5) Aligned. Score: 52.1173 
Sequences (3:6) Aligned. Score: 86.4238 Sequences (3:7) Aligned. Score: 57.2973 Sequences (3:8) Aligned. Score: 66.6667 Sequences (3: 9) Aligned. Score: 60.9375 Sequences (3:10) Aligned. Score: 56.8783 Sequences (4:5) Aligned. Score: 58.9577 Sequences (4:6) Aligned. Score: 75.4967 Sequences (4:7) Aligned. Score: 58.9189 Sequences (4:8) Aligned. Score: 56.0411 Sequences (4:9) Aligned. Score: 60.1542 Sequences (4:10) Aligned. Score: 47.8836 Sequences (5:6) Aligned. Score: 55.6291 Sequences (5:7) Aligned. Score: 65.798 Sequences (5:8) Aligned. Score: 56.6775 Sequences (5:9) Aligned. Score: 71.6612 Sequences (5:10) Aligned. Score: 50.8143 Sequences (6:7) Aligned. Score: 60.596 Sequences (6:8) Aligned. Score: 65.894 Sequences (6:9) Aligned. Score: 68.2119

Sequences (6:10) Aligned. Score: 54.3046

Sequences (7:8) Aligned. Score: 70.2703

Sequences (7:9) Aligned. Score: 77.5676

Sequences (7:10) Aligned. Score: 52.7027

Sequences (8:9) Aligned. Score: 65.5172

Sequences (8:10) Aligned. Score: 61.9048

Sequences (9:10) Aligned. Score: 60.582

As per the above score and the phylogenetic tree (Figure 2), it was clear that though, Drosophila ananesse, Drosophila melanogaster, Drosophila jambulina and Bactrocera cucurbitae belonged to same order Diptera, all the species of Drosophila in terms of partial 16SrRNA gene sequence were evolutionary more close to Dysdercus

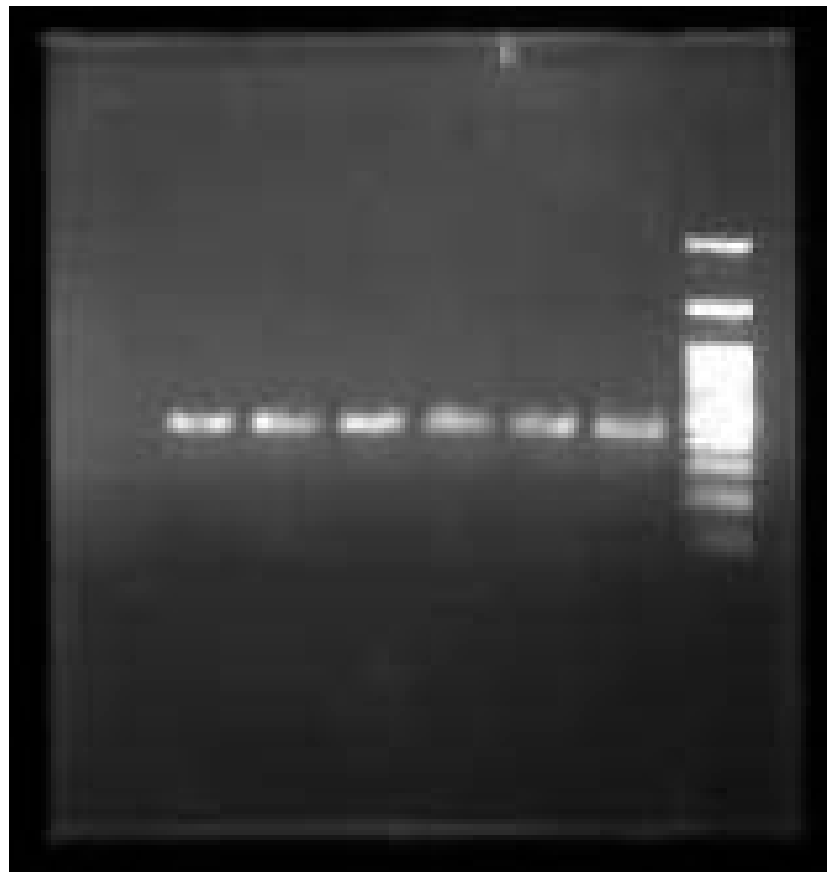

Figure 1. Distinct amplification of samples approximately corresponds to the $500 \mathrm{bp}$ of the DNA marker (extreme right). 
koenigii of order Heteroptera than Bactrocera cucurbitae. Bactrocera cucurbitae was placed in different branch and Dysdercus koenigii was placed in same branch of phylogenetic tree with respect to all species of Drosophila. Similarly, Spodoptera litura, Helicoverpa armigera and Pieris brassicae belonged to same order Lepidoptera. Spodoptera litura and Helicoverpa armigera belonged to same family Noctuidae whereas Pieris brassicae belonged to family Pieridae. The results showed that Spodoptera litura in terms of partial 16SrRNA gene sequence was evolutionary more close to Pieris brassicae. Spodoptera litura and Pieris brassicae were placed in same branch whereas Helicoverpa armigera was placed in different branch of the phylogenetic tree. Although we could not judge the sequence similarities by studying a partial sequence of 16SrRNA, this finding was unexpected as it was expected that genera of same orders and families were evolutionary close to each other. Similar results were showed by Shouche et al. [10], where mitochondrial 16SrRNA gene fragment was analyzed in mosquito. A phylogenetic tree was displayed and it showed that all studied species of Aedes, Culex and Anopheles belonging to same order Diptera and same family Culicidae were not in the same branch and thus might have had different evolutionary origins.

\section{Conclusion}

Insects having same taxonomic group might have different evolutionary origin in respect of a partial conserved gene, 16SrRNA.

Table 3. Name of the organism with accession numbers.

\begin{tabular}{crc}
\hline S.no. & Name of the Organism & Accession Numbers \\
\hline 1 & Drosophila ananesse & JX896435 \\
2 & Drosophila melanogaster & JX896436 \\
3 & Drosophila jambulina & JX912717 \\
4 & Bactrocera cucurbitae & JX912718 \\
5 & Coccinella septempunctata & JX896437 \\
6 & Dysdercus koenigii & JX974556 \\
7 & Spodoptera litura & KC352618 \\
8 & Helicoverpa armigera & KC352619 \\
9 & Pieris brassicae & KC978889 \\
10 & Apis mellifera & KC422452 \\
\hline
\end{tabular}

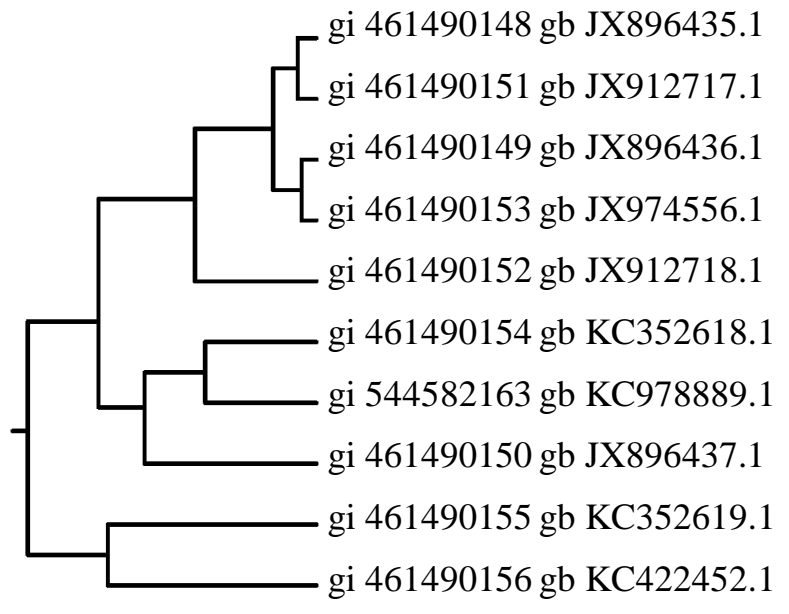

Figure 2. Phylogenetic tree of the above sequences based on rooted and branched clustal W software. 


\section{Acknowledgements}

The corresponding author is thankful to DST for funding (DST Fast Track Project).

\section{References}

[1] Adoutte, A., Balavoine, G., Lartillot, N. and Rosa, R. (1999) Animal Evolution. The End of the Intermediate Taxa? Trends in Genetics, 15, 104-108. http://dx.doi.org/10.1016/S0168-9525(98)01671-0

[2] Xiang, B. and Kochar, T.D. (1991) Comparison of Mitochondrial DNA Sequences of Seven Morphospecies of Black Flies (Diptera: Simuliidae). Genome, 34, 306-311. http://dx.doi.org/10.1139/g91-050

[3] Kambhampati, S. (1995) A Phylogeny of Cockroaches and Related Insects Based on DNA Sequence of Mitochindrial Ribosomal RNA Genes. Proceedings of the National Academy of Sciences of the United States of America, 92, 20172020. http://dx.doi.org/10.1073/pnas.92.6.2017

[4] Tang, J., Pruess, K., Cupp, E.W. and Unnasch, T.R. (1996) Molecular Phylogeny and Typing of Blackflies (Diptera: Simuliidae) That Serve as Vectors of Human or Bovine Onchocerciasis. Medical and Veterinary Entomology, 10, 228234. http://dx.doi.org/10.1111/j.1365-2915.1996.tb00735.x

[5] Tang, J. and Unnasch, T.R. (1997) Heteroduplex Analysis in Medical Entomology: A Rapid and Sensitive Sequence Based Tool for Population and Phylogenetic Studies. Parasitology Today, 13, 271-274. http://dx.doi.org/10.1016/S0169-4758(97)01062-4

[6] Brown, W.M., Matthew Jr., G. and Wison, A.C. (1979) Rapid Evolution of Mitochondrial DNA. Proceedings of the National Academy of Sciences of the United States of America, 76, 1967-1971. http://dx.doi.org/10.1073/pnas.76.4.1967

[7] Misof, B., Anderson, C.L. and Hadrys, H. (2000) A Phylogeny of the Damselfly Enuscalopteryx (Odonata) Using Mitochondrial 16SrDNA Markers. Medical and Veterinary Entomology, 15, 5-14. http://dx.doi.org/10.1006/mpev.1999.0724

[8] Crozier, R.H. and Crozier, Y.C. (1993) The Mitochondrial Genome of the Honeybee Apis mellifera: Complete Sequence and Genome Organization. Genetics, 133, 97-117.

[9] Dowton, M. and Austin, A.D. (1994) Molecular Phylogeny of the Insect Order Hymenoptera: Apocritan Relationships. Proceedings of the National Academy of Sciences of the United States of America, 91, 9911-9915. http://dx.doi.org/10.1073/pnas.91.21.9911

[10] Yogesh, S.S. and Milind, P.S. (2000) Sequence Analysis of Mitochondrial 16S Ribosomal RNA Gene Fragment from Seven Mosquito Species. Journal of Biosciences, 25, 361-366. http://dx.doi.org/10.1007/BF02703789 